\title{
SENSITIVITY PATTERN OF AZITHRYMYCIN, OFLOXACIN AND CEFTRIAXONE IN CIPROFLOXACIN RESISTANT SALMONELLA CAUSING ENTERIC FEVER
}

\author{
KAWSER $\mathrm{S}^{1}$, MIAH MRA ${ }^{2}$, SABAH KMN ${ }^{3}$, BEGUM T $^{4}$, SULTAN $\mathrm{S}^{5}$
}

\begin{abstract}
The therapeutic alternatives available for use against ciprofloxacin resistant enteric fever isolates in an endemic area are limited. A cross sectional study was carried out in the Department of Microbiology \& Immunology, Bangabandhu Sheikh Mujib Medical University (BSMMU), Dhaka, from January to December 2008, to see the sensitivity pattern of azithrymycin, ofloxacin and ceftriaxone in ciprofloxacin resistant salmonella causing enteric fever. In this study, the MICs of various drugs were determined for 100 enteric fever isolates (72 Salmonella enterica serovar Typhi and 28 Salmonella enterica serovar paratyphi A). By agar dilution method, 40\% Salmonella strains were ciprofloxacin resistant showing MIC values of $(4-8 \mathrm{mg} / \mathrm{ml}), 36 \%$ strains were intermediate sensitive with MIC values of 1 to $4 \mathrm{mg} / \mathrm{ml}$ and $24 \%$ strains were sensitive to ciprofloxacin showing MIC values of 0.125 to $1 \mathrm{mg} / \mathrm{ml}$. All ciprofloxacin-resistant isolates were sensitive to ofloxacin (inhibitory zone diamater 16-32 $\mathrm{mm}$ ), ceftriaxone (inhibitory zone diameter $21 \mathrm{~mm}), 66.66 \%$ isolates were sensitive to azithromycin. These results indicate that ofloxacin and ceflriaxone may be convenient alternative antimicrobial agents for Salmonella isolates.
\end{abstract}

Key words: Enteric fever, Sensitivity pattern, Ciprofloxacin resistant salmonella

J Dhaka Med Coll. 2013; 22(1) : 55-60.

\section{Introduction}

Enteric fever caused by Salmonella Typhi \& Salmonella Paratyphi A.B.C . ${ }^{1}$ Enteric fever due to infection with Salmonella enterica serovar Typhi or Salmonella enterica serovar Paratyphi A is estimated to cause more than 27 million infections each year worldwide with 216000 deaths. ${ }^{2}$ After extensive outbreaks of typhiod fever occurred in Mexico and India in the early \& mid 1970s, in which epidemic strains were resistant to chloramphenicol, the efficacy of this antimicrobial was in doubt. Alternative drugs for typhoid fever are ampicillin and trimethoprim. However, following outbreaks in the Indian subcontinent, the Arabian Gulf, the Philippines and South Africa in the late 1980s and early 1990s, in which causative strains were resistant to ampicillin and trimithoprim in addition to chloramphenicol, the efficacy of these antimicrobials has also been impaired. ${ }^{3}$
In the last two decades, the worldwide emergence of multi-drug resistant strains of Salmonella has led to virtual withdrawal of chloramphenicol and its replacement with fluoroquinolones and third generation cephalosporins. Clinical treatment failures after the administration of ciprofloxacin and other fluoroquinolones to patient with typhoid fever attributable to these strains have been reported. The emergence of complete resistant to ciprofloxacin in S. Typhi or S. Paratyphi A would severely limit the choice of antimicrobial therapy for treating enteric fever. ${ }^{4}$ Where fluoroquinolones, such as ciprofloxacin and ofloxacin, have become widely used, isolates of Salmonella enterica serovar Typhi and serovar Paratyphi A with reduced susceptibility to fluoroquinolones have become common. ${ }^{2}$ The most common method of testing for resistance

1. Dr. Shamima Kawser, Assistant Professor, Department of Microbiology, Delta Medical College, Dhaka.

2. Prof. Md. Ruhul Amin Miah, Chairman, Department of Microbiology \& Immunology, Bangabandhu Sheikh Mujib Medical University (BSMMU), Dhaka.

3. Dr. Khandker Md. Nurus Sabah, Assistant Professor, Department of Cardiology, Dhaka Medical College, Dhaka.

4. Dr. Tanzima Begum, Assistant Professor, Department of Pharmacology \& Therapeutics, Delta Medical College, Dhaka.

5. Dr. Shahin Sultana, Associate Professor, Department of Microbiology, Delta Medical College, Dhaka.

Correspondence : Dr. Shamima Kawser, Assistant Professor, Department of Microbiology, Delta Medical College, Dhaka. Cell Phone: +8801715495203, E-mail: sabahkmn@yahoo.com 
to ciprofloxacin in clinical laboratories is by disc diffusion, using discs with concentration of ciprofloxacin ranging from 1 to $5 \mathrm{mg} / \mathrm{L}^{5}$

However, isolates of Salmonella enterica serovars Typhi and Paratyphi A with reduced susceptibility to fluoroquinolones (as indicated in the laboratory by resistance to nalidixic acid) have now appeared in the Indian subcontinent, Vietnam and Tajikistan and treatment failures with fluoroquinolones have also been reported. Isolates of serovar Typhi in the United Kingdom had reduced susceptibility to ciprofloxacin. ${ }^{6}$ In order to effectively monitor the changing trends in the level of antimicrobial for testing the susceptibility of Salmonella Typhi strains to selected antibiotics should be used routinely in large hospital settings in more geographical areas. ${ }^{7}$ A national-guide line on the proper usage of antibiotics is required for urgent implementation in Bangladesh. ${ }^{8}$ In the present study, sensitivity pattern of this four drugs including nalidixic acid was determined by disc diffusion method.

\section{Methods}

This cross sectional study was carried out in the department of Microbiology \& Immunology, Bangabandhu Sheikh Mujib Medical University (BSMMU), Dhaka, from January to December 2008. 100 samples of isolated Salmonella were collected from Microbiology \& Immunology Laboratory of BSMMU, Dhaka. Isolated Salmonella was collected from Microbiology \& Immunology laboratory of BSMMU which was subsequently subcultured and confirmed by biochemical test. Specimens were preserved at $2-8^{\circ} \mathrm{C}$ in nutrient agar slant. Antimicrobial sensitivity test was done for all Salmonella isolates with five antimicrobial agents (Ciprofloxacin, Ofloxacin, Ceftriaxone, Azithromycin \& Nalidixic acid) using MuellerHinton agar medium. The sensitivity test includes disc diffusion method to find out inhibitory zone diameters to the disc of recommended strength

Disc diffusion method of antimicrobial sensitivity test:

Disc of the antimcrobial agents used in different strengths. Mueller-Hinton agar medium was used for disc diffusion test. A suspension of each isolate was made in sterile nutrient broth. At least three to five wellisolated colonies of the same morphological type was selected from an agar plate culture. The top of each colony was touched with a loop and the growth was transferred into a tube containing 4-5 $\mathrm{ml}$ of sterile nutrient broth. The turbidity of the actively growing broth culture was adjusted with that of the $0.5 \mathrm{McF}$ arland standard, which is equivalent to $10^{7} \mathrm{CFU} / \mathrm{ml}$. The dried surface of a Mueller-Hinton agar plate was inoculated by streaking the swab over the entire sterile agar surface. The disc were placed over the inoculated surface about 15-20 $\mathrm{mm}$ away from the edge of the petridish and $24 \mathrm{~mm}$ from center to center to prevent overlapping of zones of inhibition. In this method, a maximum of 5 discs were placed into a $90 \mathrm{~mm}$ plate. After placing the antimicrobial disc, the inoculated plates were incubated at $37^{\circ} \mathrm{C}$ for $18-24$ hours. Then the diameters of the zones of inhibition were measured in millimeter. The interpretive criteria to evaluate the susceptibility of the isolates were done according to Cultural Laboratory Standard Institute (CLSI) guideline.

\section{Results}

A total of 100 Salmonella strains were studied, of which $72(72 \%)$ were Salmonella Typhi, 28 $(28 \%)$ were S. Paratyphi A and none was S. Paratyphi B. Sensitivity pattern of ciprofloxacin, ofloxacin, ceftriaxone azithromycin and nalidixic acid among salmonella species by disc diffusion method was given in (Table-I). 75 (75\%) sensitive, 22 (22\%) intermediately sensitive and $3(3 \%)$ salmonella strains were resistant to ciprofloxacin. 100 $(100 \%)$ strains were sensitive to ofloxacin. 94 $(94 \%)$ strains were sensitive and $6(6 \%)$ strains were intermediately sensitive to ceftriaxone. $71(71 \%)$ strains were sensitive and 29 (29\%) strains were intermediately sensitive to azithromycin. Again, 90 (90\%) strains were resistant and $10(10 \%)$ were sensitive to nalidixic acid. Table-II shows that out of 90 nalidixic acid resistant strains of Salmonella, $66(73.33 \%)$ were sensitive, 21 (23.33\%) were intermediate sensitive and 3 (3.33\%) salmonella strains were resistant to 
ciprofloxacin. Among 10 nalidixic acid sensitive strain, $8(80 \%)$ were sensitive and 2 $(20 \%)$ were resistant to ciprofloxacin. Sensitivity pattern of ofloxacin, ceftriaxone and azithromycin among three ciprofloxacin resistant salmonella species by disc diffusion method was shown in (Table-III, IV \& V). All 3 ciprofloxacin resistant strains were sensitive to ofloxacin and ceftriaxone. 2 (66.66\%) salmonella strains were sensitive and 1 $(33.33 \%)$ strain was intermediate sensitive to azithromycin. All 22 ciprofloxacin intermediate sensitive salmonella strains were sensitive to ofloxacin, $21(95.45 \%)$ strains were sensitive and $1(4.55 \%)$ strains intermediate sensitive to ceftriaxone. $11(50 \%)$ strains were sensitive and 11 (50\%) salmonella strains were intermediate sensitive to azithromycin. All 75 ciprofloxacin sensitive salmonella strains were sensitive to ofloxacin. 70 (93.33\%) strains were sensitive and $5(6.66 \%)$ strains were intermediate sensitive to ceftriaxone. 57 (76\%) strains were sensitive and $18(24 \%)$ strains were intermediate sensitive to azithromycin.

\section{Table-I}

Sensitivity pattern of ciprofloxacin, ofloxacin, ceftriaxone, azithromycin and nalidixic acid among Salmonella species by disc diffusion method $(n=100)$

\begin{tabular}{lccc}
\hline \multirow{2}{*}{$\begin{array}{l}\text { Name of antimicrobial } \\
\text { agents }\end{array}$} & \multicolumn{3}{c}{ No. of isolates } \\
\cline { 2 - 4 } & Resistant & $\begin{array}{c}\text { Intermediate } \\
\text { sensitivity }\end{array}$ & Sensitive \\
\hline Ciprofloxacin & $3(3.0 \%)$ & $22(22.0 \%)$ & $75(75.0 \%)$ \\
Ofloxacin & $0(0.0 \%)$ & $0(0.0 \%)$ & $100(100 \%)$ \\
Ceftriaxone & $0(0.0 \%)$ & $6(6.0 \%)$ & $94(94.0 \%)$ \\
Azithromycin & $0(0.0 \%)$ & $29(29.0 \%)$ & $71(71.0 \%)$ \\
Nalidixic acid & $90(90.0 \%)$ & $0(0.0 \%)$ & $10(10.0 \%)$ \\
\hline
\end{tabular}

Table- II

Sensitivity pattern of ciprofloxacin in relation to sensitivity pattern of nalidixic acid by disc diffusion test $(n=100)$

\begin{tabular}{lccc}
\hline Nalidixic acid & \multicolumn{3}{c}{ Ciprofloxacin } \\
\cline { 2 - 4 } & $\begin{array}{c}\text { No. of resistant } \\
\text { strain }\end{array}$ & $\begin{array}{c}\text { No. of } \\
\text { intermediate } \\
\text { sensitive strain }\end{array}$ & $\begin{array}{c}\text { No. of sensitive } \\
\text { strain }\end{array}$ \\
\hline Resistant strain $(\mathrm{n}=90)$ & $3(3.3 \%)$ & $21(23.33 \%)$ & $66(73.33 \%)$ \\
Sensitive strain $(\mathrm{n}=10)$ & 0 & $2(20.0 \%)$ & $8(80.0 \%)$ \\
\hline
\end{tabular}

Table-III

Sensitivity pattern of ofloxacin, ceftriaxone and azithromycin among ciprofloxacin resistant Salmonella species by disc diffusion method $(n=3)$

\begin{tabular}{lccc}
\hline Antimicrobial & \multicolumn{3}{c}{ No. of isolates } \\
\cline { 2 - 4 } Agents & Resistant & $\begin{array}{c}\text { Intermediate } \\
\text { sensitivity }\end{array}$ & Sensitive \\
\hline Ofloxacin & $0(0.0 \%)$ & $0(0.0 \%)$ & $3(100.0 \%)$ \\
Ceftriaxone & $0(0.0 \%)$ & $0(0.0 \%)$ & $3(100.0 \%)$ \\
Azithromycin & $0(0.0 \%)$ & $1(33.33 \%)$ & $2(66.66)$ \\
\hline
\end{tabular}


Table-IV

Sensitivity pattern of ofloxacin, ceftriaxone and Azithromycin among ciprofloxacin intermediate susceptibility Salmonella species by disc diffusion method $(n=22)$

\begin{tabular}{lccc}
\hline $\begin{array}{l}\text { Antimicrobial } \\
\text { Agents }\end{array}$ & Resistant & $\begin{array}{c}\text { No. of isolates } \\
\text { Intermediate } \\
\text { sensitivity }\end{array}$ & Sensitive \\
\hline Ofloxacin & $0(0.0 \%)$ & $0(0.0 \%)$ & $22(100 \%)$ \\
Ceftriaxone & $0(0.0 \%)$ & $1(4.54 \%)$ & $21(95.45 \%$ \\
Azithromycin & $0(0.0 \%)$ & $11(50 \%)$ & $11(50 \%)$ \\
\hline
\end{tabular}

\section{Table-V}

Sensitivity pattern of ofloxacin, ceftriaxone and Azithromycin among ciprofloxacin sensitive Salmonella species by disc diffusion method $(n=75)$

\begin{tabular}{lccc}
\hline Antimicrobial & \multicolumn{3}{c}{ No. of isolates } \\
\cline { 2 - 4 } Agents & Resistant & $\begin{array}{c}\text { Intermediate } \\
\text { sensitivity }\end{array}$ & Sensitive \\
\hline Ofloxacin & $0(0.0 \%)$ & $0(0.0 \%)$ & $75(100 \%)$ \\
Ceftriaxone & $0(0.0 \%)$ & $5(6.66 \%)$ & $70(93.33 \%)$ \\
Azithromycin & $0(0.0 \%)$ & $18(24 \%)$ & $57(76 \%)$ \\
\hline
\end{tabular}

\section{Discussion}

In the present study, by disc diffusion method showed that only $3 \%$ salomenlla isolates were resistant to ciprofloxacin, $22 \%$ were intermediate sensitive and $75 \%$ were sensitive to ciprofloxacin. The results were in consistent with the study of Gautam et al. $(2004)^{9}$ who showed that $79 \%$ salmonella strains were sensitive to ciprofloxacin by disc diffusion method. A study by Chowta and Chowta (2005) ${ }^{10}$ showed that there was in vitro sensitive to ciprofloxacin, but the patient did not respond to the drug therapy. These findings suggest that sensitivity of salmonella to ciprofloxacin in vivo gradually decreasing. A strain with decreased sensitive to ciprofloxacin was reported from the Indian subcontinent and south east and central Asia. Disc diffusion testing revealed that these isolates were resistant to nalidixic acid but sensitive to ciprofloxacin, according to the current CLSI interpretive criteria. $^{11}$

In the present study, $90 \%$ salmonella strains were resistant and $10 \%$ were sensitive to nalidixic acid by disc diffusion method. The resistant rate to nalidixic acid in this study was significantly higher than that of Chinh et al. $(2000)^{6}$ in Vietnam, who reported only 53\% of isolates were resistant to nalidixic acid. Mendal (2004) ${ }^{12}$ from Kolkata reported 100\% salmonella strains were resistant to nalidixic acid, indicating the reduced susceptibility to ciprofloxacin. In present study, $73.33 \%$ of nalidixic acid resistant salmonella strains were sensitive, $23.33 \%$ were intermediate sensitive and $3.33 \%$ were resistant to ciprofloxacin. Among nalidixic acid sensitive salmonella strains, $80 \%$ strains were sensitive and $20 \%$ were resistant to ciprofloxacin. Therefore, nalidixic acid resistance determined by the disc diffusion method could be an indication of decreased susceptibility to ciprofloxacin. ${ }^{13}$ The presence of nalildixic acid resistance has been suggested as a laboratory marker of isolates with reduced susceptibility to fluoroquinolones and indicates that invasive infection may fail to respond to fluoroquinolone therapy. ${ }^{14} 100 \%$ salmonella strains were sensitive to ofloxacin with zone diameter ${ }^{3} 16 \mathrm{~mm}$ in this study. This finding correlates with Mandal et al. (2003) ${ }^{12}$, who found $99.04 \%$ salmonella strains were sensitive to ofloxacin by disc diffusion method with zone diameter ${ }^{3} 16 \mathrm{~mm}$. It also correlates with Choudhari and Bansal (1997) ${ }^{15}$ and Kumar et al. $(2001)^{16}$, who found to most of the strains 
were ofloxacin sensitive by disc diffusion method.

Several previous studies had observed clinical failure in response to ciprofloxacin therapy due to infection with nalidixic acid resistant salmonella isolates and many studies had considered as nalidixic acid sensitivity test as surrogate marker for decreased sensitivity to ciprofloxacin among Salmonella enterica serovar Typhi. ${ }^{17,18}$ Wain et al. (1997) ${ }^{19}$ reported similar observation regarding ofloxacin sensitivity to Salmonella enterica serovar Typhi that means treatment failure occured to ofloxacin therapy if infection occured with nalidixic acid resistant Salmoella isolates. The present study showed that, $100 \%$ ciprofloxacin resistant salmonella strains were sensitive to ofloxacin. A study done by Capoor et al. (2006) 20 showed that variation in result between first and second generation quinolones, $96.2 \%$ of strains resistant for ofloxacin and $92.3 \%$ for levofloxacin. These observations indicate that fluoroquinolones should be tested individually and the ciprofloxacin not represent this group adequately Kumar et al. (2002). ${ }^{16}$ Disc diffusion method of sensitivity testing of salmonella to ceftriaxone showed that $94 \%$ strains were ceftriaxone sensitive. This result is not consistent with the result of Gautom et al. $(2002)^{8}$, who showed $88 \%$ S. Typhi were sensitive to ceftriaxone by disc diffusion method. Azithromycin in the present study showed that $29 \%$ of salmonella isolates were intermediate sensitive to azithromycin with zone diameter 14-17 mm with MIC 2-64 mg/ $\mathrm{ml}$ and $71 \%$ of isolates were sensitive to azithromycin by disc diffusion method had zone diamerter ${ }^{3} 18 \mathrm{~mm}$. In enteric fever, the role of azithromycin needs to be appreciated, as it is highly effective in removing intracellular salmonella, defervescence is rapid, gastrointestinal carriage is eradicated and it represents a potential alternative in pediatric populations where quinolones are contraindicated. ${ }^{21}$

\section{Conclusion}

This study has been revealed that most of the Salmonella typhi isolates which are resistant to nalidixic acid will also be resistant to ciprofloxacin. However, they may show in vitro susceptibility. For this reason, it is essential to find out a therapeutic alternative against this resistant organism.

\section{References}

1. Cheesbrough M. District laboratory practice in tropical countries. Part-II. $2^{\text {nd }}$ ed. Cambridge: Cambridge University Press; 2006.

2. Parry CM, Ho VA, Phuong LT, Bay PVB, Lanh MN, Tung LT, et al. Randomized controlled comparison of ofloxacin, azithromycin, and an ofloxacin-azithromycin combination for treatment of multidrug-resistant and nalidixic acid-resistant typhoid fever. Antimicrob Agents Chemother 2007; 51(3): 819-25.

3. Threlfall EJ, Ward LR. Decreased susceptibility to ciprofloxacin in Salmonella enterica serotype typhi, United Kingdom. Emerg Infect Dis 2001; $7(3)$ : 448-50.

4. Gaind R, Paglietti B, Murgia M, Dawar R, Uzzau $\mathrm{S}$, Cappuccinelli $\mathrm{P}$, et al. Molecular characterization of ciprofloxacin-resistant Salmonella enterica serovar typhi and paratyphi a causing enteric fever in India. J Antimicrob Chemother 2006; 58(6): 1139-44.

5. Jevanand HR, Ragavan PUM, Gunapathi RS. Study of R-factors among multidrug resistant Salmonella Typhi. Indian J Med Microbiol 1997; 15(1): 37-9.

6. Chinh NT, Parry CM, Ly NT, HA HD, Thong MX, Diep TS, et al. A randomized controlled comparison of azithromycin and ofloxacin for treatment of multidrug-resistant of nalidixic acidresistant enteric fever. Antimicrob Agents Chemother 2000; 44(7): 1855-9.

7. Mehta G, Randhawa VS, Mohapatra NP. Intermediate susceptibility to ciprofloxacin in Salmonella Typhi strains in India. Eur J Clin Microbiol Infect Dis 2001; 20: 760-1.

8. Ahmed D, Costa LT, Alam K, Nair GB, Hossain MA. Multidrug-resistant Salmonella enterica Serovar Typhi isolates with high-level resistance to ciprofloxacin in Dhaka, Bangladesh. Antimicrob Agents Chemother 2006; 44(7): 1855-9.

9. Gautam V, Gupta NK, Chaudhary U, Arora DR. Sensitivity pattern of Salmonella serotypes in northern India. Braz J Infect Dis 2002; 6(6): 14138670 .

10. Chowta MN, Chowta NK. Study of clinical profile and antibiotic response in typhoid fever. Indian J Med Microbiol 2005; 23(2): 125-7.

11. Threlfall EJ, Ward LR, Skinner JA, Smith HR, Lacey S. Ciprofloxacin resistant Salmonella Typhi and treatment failure. Lancet 1999; 353: 1590-1. 
12. Mandal S, Mandal MD, Pal NK. Ofloxacin minimum inhibitory concentration versus disk diffusion zone diameter for Salmonella enterica serovar Typhi isolates: problems in the detection of ofloxacin resistance. Jpn J Infect Dis 2003; 56: $210-2$.

13. Asna SMZ, Haq JA, Rahman MM. Nalidixic acidresistant Salmonella enterica serovar Typhi with decreased susceptibility to ciprofloxacin caused treatment failure: a report from Bangladesh. Jpn J Infect Dis 2003; 56: 32-3.

14. CLSI. Performance Standards for Antimicrobial Susceptibility Testing. $16^{\text {th }}$ information supplement, M100-S16. Wayne, PA: Clinical and Laboratory Standards Institute. 2006.

15. Choudhari A, Bansal M. Multidrug resistant Salmonella typhi in Aurangabad. Indian J Med Res 1997; 15: 155.

16. Kumar R, Aneja KR, Punia AK, Roy P, Sharma M, Gupta $R$, et al. Changing pattern of biotypes phage types and drug resistance of Salmonella Typhi in Ludhiana drug 1980-1999. Indian J Med Res 2001; 113: 175-80.
17. Kapil A, Das R, Das B. Nalidixic acid susceptibility test to screen ciprofloxacin resistance in Salmonella Typhi. Indian J Med Res 2002; 115: 49-54.

18. Hakanen A, Kotilainen P, Jalava J, Shtonen A, Huovinen P. Detection of decreased fluoroquinolone susceptibility in Salmonellas and validation of nalidixic acid screening test. J Clin Micrbiol 1999; 37: 3572-7.

19. Wain J, Hoa NTT, Chinh NT, Vinh H, Everett MJ, Diep TS, et al. Quinolone-resistant Salmonella typhi in Vietnam: molecular basis of resistance and clinical response to treatment. Clin Infect Dis 1997; 25: 1404-10.

20. Capoor MR, Nair D, Hasan AS, Aggarwal P, Gupta B. Narrowing therapeutic options in typhoid fever, India. Southeast Asian J Trop Med Public Health 2006; 37: 1170-4.

21. Girgis NI, Butler T, Frenck RW, Sultan Y, Brown FM, Tribble D, et al. Azithromycin versus ciprofloxacin for treatment of uncomplicated typhoid fever in a randomized trial in Egypt that included patients with multidrug resistance. Animicrob Agents Chemother 1999; 43: 1441-4. 